\title{
Prediction of petroleum exploration risk and subterranean spatial distribution of hydrocarbon accumulations
}

\author{
Xie Hongbing1, 2*, Guo Qiulin², Li Feng³, Li Jianzhong², Wu Na², Hu Suyun² \\ and Liang Kun ${ }^{2}$ \\ ${ }^{1}$ School of Energy Resources, China University of Geosciences, Beijing 100083, China \\ ${ }^{2}$ Research Institute of Petroleum Exploration \& Development, PetroChina, Beijing 100083, China \\ ${ }^{3}$ Science \& Technology Management Department, China National Petroleum Corporation, Beijing 100007, China \\ (C) China University of Petroleum (Beijing) and Springer-Verlag Berlin Heidelberg 2011
}

\begin{abstract}
Investigation of spatial distribution of oil and gas resource and accurate prediction of the geographic location of its undiscovered resource is significant for reducing exploration risk and improving exploration benefit. A new method for predicting spatial distribution of oil resource is discussed in this paper. It consists of prediction of risk probability in petroleum exploration and simulation of hydrocarbon abundance.

Exploration risk probability is predicted by multivariate statistics, fuzzy mathematics and information processing techniques. A spatial attribute database for sample wells was set up and the Mahalanobis distance and Fuzzy value of given samples were obtained. Then, the Bayesian formula was used to calculate the hydrocarbon-bearing probability at the area of exploration wells. Finally, a hydrocarbon probability template is formed and used to forecast the probability of the unknown area.

The hydrocarbon abundance is simulated based on Fourier integrals, frequency spectrum synthesis and fractal theory. Firstly, the fast Fourier transformation (FFT) is used to transform the known hydrocarbon abundance from the spatial domain to the frequency domain, then, frequency spectrum synthesis is used to produce the fractal frequency spectrum, and FFT is applied to get the phase information of hydrocarbonbearing probability. Finally, the frequency spectrum simulation is used to calculate the renewed hydrocarbon abundance in the play.

This method is used to predict the abundance and possible locations of the undiscovered petroleum accumulations in the Nanpu Sag of the Bohai Bay Basin, China. The prediction results for the wellexplored onshore area of the northern Nanpu Sag agree well with the actual situations. For the lessexplored offshore areas in the southern Nanpu Sag, the prediction results suggest high hydrocarbon abundance in Nanpu-1 and Nanpu-2, providing a useful guiding for future exploration.
\end{abstract}

Key words: Hydrocarbon resources, abundance, risk forecast, Nanpu sag, frequency spectrum simulation, fractal theory

\section{Introduction}

In the last 40 years, hydrocarbon resource assessment has been focused on the total resource and its distribution in an exploration area, and many methods and techniques have been developed, such as the geological analogy method, Delphi forecasting method or subjective discrimination method, historical performance or features extrapolated method, and various geological and statistical model methods (Zhao et al, 2005). In contrast, only a few studies have focused on spatial

*Corresponding author. email: xhb@petrochina.com.cn Received December 30, 2009 characteristics of oil and gas accumulations, accordingly, few prediction methods were developed. In the last 10 years, a few methods have been developed for predicting the geographic location of undiscovered resources. For example, Gao et al (2000) developed a object-based stochastic approach, Chen et al (2002) and Chen and Osadetz (2006b) developed a random modeling method by using Fourier transformation to forecast oil and gas spatial distribution. To date, with the petroleum industry growth, prediction of spatial distribution of hydrocarbon, as a supplement and extension of the traditional assessment methods, attracts increasingly attention of oil companies and researchers.

In this paper, we discuss a new method for predicting 
oil and gas spatial distribution. It consists of prediction of exploration risk probability and simulation of hydrocarbon abundance. The former is to predict the hydrocarbons-bearing probability by multivariate statistics, fuzzy mathematics and information processing technique, and obtain a hydrocarbonbearing probability map through quantitative calculation to show the exploration risk in the area. Subsequently, the latter (i.e. simulation of hydrocarbon abundance) is to integrate the information on exploration risk probability, exploration results and other data at the frequency domain for analysis and processing, and then transform them to the spatial domain to get a hydrocarbon abundance map. In this way, the spatial distribution of hydrocarbons and size of reservoirs can be predicted quantitatively.

\section{Prediction of risk probability in oil and gas exploration}

Prediction of exploration risk probability is to quantitatively judge the type of undiscovered area (Chen and Osadetz, 2006a). All the exploration wells are integrated as a gather and classified into two subset groups-oil wells group and dry holes group. Moreover, a characteristic database composed of geological characteristic variables is established to reflect the two subset groups. By comparing the undiscovered area with the two subset groups, and by using multivariate statistics, fuzzy comprehensive discrimination and random modeling, we can calculate the hydrocarbonbearing probability of wells to be drilled in future at the position (Chen et al, 2002; Chen and Osadetz, 2006a; Hu et al, 2007; Guo et al, 2007; Hu et al, 2009).

To show the method of predicting exploration risk, in this paper the exploration risk was analyzed for the 1st Member of Dongying Formation $\left(\mathrm{Ed}_{1}\right)$ of the Tertiary succession in the Nanpu Sag in the Bohai Bay Basin, China. The principles and details of the method have been reported by Chen and Osadetz (2006a) and Hu et al (2007; 2009). The processes of prediction of risk probability in oil and gas exploration are as follows.

(1) Analysis of oil and gas accumulation rules in sag

Based on concluding the distribution rule of oil and gas accumulation in different plays in the Bohai Bay Basin, with secondary structural belts as units, and considering the double-faulted structure of the Nanpu Sag, basically investigate the sedimentary system, characteristics of reservoir development, connectivity system and hydrocarbon accumulation characteristics.

\section{(2) Establishment of the evaluation parameter system}

Setting up the evaluation parameter system and value criteria for the 1st Member of the Dongying Formation $\left(\operatorname{Ed}_{1}\right)$ in the Nanpu Sag by subdividing the member in terms of oil source, reservoir and cap matching condition, trap condition and so on.

\section{(3) Test of the validity of parameters}

Not all the geological parameters are valid, so their validity must be tested. For the Mahalanobis distance discrimination method, only valid ones are selected. For the fuzzy comprehensive discrimination method, the parameters that can identify the two subset groups are weighted with high value; otherwise they are weighted with low value.

(4) Establishment of the spatial characteristic database for oil and gas wells and dry holes

Establishing the spatial characteristic database for the related target layer, by re-identifying and analyzing the major geological factors controlling oil and gas distribution in $\mathrm{Ed}_{1}$ in the Nanpu sag.

(5) Establishment of the prediction template

The Mahalanobis distance (MD) discrimination and fuzzy mathematics methods are used to establish a prediction template of hydrocarbon-bearing probability. The hydrocarbon-bearing probability is calculated for each well by using a Bayesian approach.

(6) Prediction of risk probability for petroleum exploration and analysis of the prediction results

The Mahalanobis distance and fuzzy value are transformed into hydrocarbon-bearing probability according to the prediction templates; then the spatial distribution of hydrocarbon accumulations can be interpolated by a Kriging interpolation method, and the visualization technique is used to obtain an MD hydrocarbon-bearing probability map, fuzzy discrimination hydrocarbon-bearing probability map and comprehensive hydrocarbon-bearing probability map, on these maps the points with high probability mean low-risk points for petroleum exploration. In this way, the exploration risk in the whole area are revealed.

\section{Simulation of hydrocarbon abundance}

Simulation of hydrocarbon abundance consists of fractal simulation and conditional simulation. The fractal simulation is to identify the spatial characteristics of small oil/gas fields according to the spatial characteristics of discovered large oil/ gas fields (Chen et al, 2002; Chen and Osadetz, 2006b), based on the scaling property of a fractal model, and considering the feature of petroleum exploration, that is, larger fields are discovered early in exploration process (Kaufman et al, 1975; Scheunemeyer and Drew, 1983). The conditional simulation is derived from the concept proposed by Chen and Osadetz (2006b). We use a Fourier integral approach for the conditional simulation. A modification of the phase identification approach reported by Yao (1998a; 1998b) can use indirect soft-information from study of the petroleum system to constrain the location of predicted resources.

Firstly, fractal simulation is conducted to forecast the size and spatial characteristics of reservoirs; then, conditional simulation is used to integrate the exploration risk probability, exploration results and other geological data, and finally the quantitative prediction of spatial distribution and reservoir size is completed.

\subsection{A fractal model for petroleum accumulation}

Barton and Scholz (1995) and La Pointe (1995) studied the data from well-explored sedimentary basins in the United States and concluded that the size and spatial distribution of hydrocarbon accumulations is fractal. The study of Western Canada Sedimentary Basin (WCSB) by Chen et al (2001) and the study of the Nanpu Sag and large-sized oil fields in China by Guo et al (2009) also indicate similar fractal characteristics 
of petroleum accumulations. Furthermore, Chen et al (2002) proposed a power spectrum representation of a fractal model for petroleum accumulations. The scaling property of a fractal model implies that the spatial characteristics of large petroleum accumulations could be used to infer the spatial characteristics of the small accumulations, which are typically underrepresented in an exploration data set. Thus, the spatial characteristics of petroleum accumulations can be inferred from the biased observation resulting from exploration (Chen and Osadetz, 2006b).

\subsection{Power spectrum representation of fractal model}

Several methods are available to generate fractal images, such as Poisson faulting, random mid-point displacement, Progressive Random Increment, Fourier Transformation (or Frequency Spectrum Synthesis) and Wavelet Conversion methods. Fourier Transformation is a popular mathematical approach for signal and image processing, which can transform an image from the spatial domain to the frequency domain (in other words, the spectrum distribution of original image, and it includes amplitude spectrum and phase spectrum; the square of amplitude spectrum is called power spectrum), or conversely it can retransform the frequency domain to the original spatial domain. It is suitable for $2 \mathrm{D}$ image generation due to its two advantages: (1) convenient and fast computation (by using appropriate FFT arithmetic); and (2) it is possible to separately study the spatial correlations and location characteristics by frequency spectrum analysis. By using the Fourier transformation, the spatial distribution, locations and size of hydrocarbon accumulations can be conveniently extracted and analyzed from different geological data sources and subsequently integrated in the simulation in a frequency domain.

For a fractal time series, the power spectrum function is expressed as a power function of the time series frequency (Turcotte, 1997):

$$
S(f) \propto \frac{1}{f^{\beta}}
$$

where $f$ is the frequency, and $\beta$ is an exponential coefficient.

In the fractal model, the spatial correlation of objects is fully specified by the logarithmically transformed spectrum density function. In general, the stochastic processes in Eq. (1) represents a fractional Brownian motion ( $\mathrm{fBm})$ with $H=(\beta \quad \square \| \square: \mathrm{KHQ} \beta$ is in the range of $1-3$, it generates a fBm with a fractal dimension $D_{f} \square H \square \beta$ ) $/ 2$. Let $u$ and $v$ represent the frequencies in $x$ and $y$ directions, then the power spectrum $S(u, v)$ of a $2 \mathrm{D} \mathrm{fBm}$ depends only on $\sqrt{u^{2}+v^{2}}$. In an isotropic case, the $2 \mathrm{D}$ process can be inferred from Eq. (1) as (Saupe, 1988):

$$
S(u, v)=\frac{1}{\left(u^{2}+v^{2}\right)^{H+1}}
$$

In an anisotropic case, by defining $H$ as a function of azimuth $\theta$, we have:

$$
S(u, v)=\frac{1}{\left(u^{2}+v^{2}\right)^{H(\theta)+1}}
$$

Eqs. (2) and (3) represent the fractal models of petroleum resource. The discovered petroleum accumulations and exploration data can be used to infer the fractal parameters. Given reservoir data, the models can be used for simulation, revising the amplitude spectrum of petroleum accumulations and eliminating the effect of sampling bias on the prediction results, so as to better describe the size and spatial distribution of reservoirs.

\subsection{Conditional simulation}

In this paper, the algorithm of conditional simulation for undiscovered petroleum accumulations is based on Yao's phase identification approach (Yao, 1998a;1998b) with some modifications of it (Xie et al, 2007). The core idea of the method is adopting the phase of the hydrocarbon-bearing probability map obtained from the exploration risk analysis to determine the locations of undiscovered petroleum resources in phase iterative identification (Chen and Osadetz, 2006b). The phase identification approach only considers the hard data from the previous exploration drilling results at the data points for conditioning. The algorithm used in this study also considers the soft information in a probability map of petroleum accumulation, integrating all available geological information in the study area.

A petroleum resource map (an image) was generated by using the discovered petroleum accumulations and dry holes locations. Resource abundances are assigned to the cells occupied by discovered hydrocarbon accumulations and value " 0 " assigned to the dry holes location and their influencing zones. The petroleum resource map is then transformed into a frequency domain using fast Fourier transform (FFT), obtaining an amplitude map and phase map. The fractal model is used to generate an unbiased estimation of the model parameters, to obtain a new amplitude map. The iteration process of the simulation is as follows:

Step 1: Inverse FFT is applied to the new amplitude map and the original phase map to produce an unconditional simulation, $Z_{\mathrm{s}}$;

Step 2: Compute the differences of resource abundance between the simulated $Z_{\mathrm{s}}$ and observed $Z\left(i_{a}, j_{a}\right), a=1,2$, $\cdots, n$ at every data point. The sum of the differences is the objective function, which will be minimized.

$$
o b j=\sum_{a=1}^{n}\left|\frac{z_{s}\left(i_{a}, j_{a}\right)-z\left(i_{a}, j_{a}\right)}{z\left(i_{a}, j_{a}\right)}\right|
$$

Step 3: If the objective function obj, in Eq. (4), is less than a pre-set tolerance value $o b j_{\text {min }}$, stop the simulation and accept the image as the final resource map. Otherwise replace the simulated values with conditional value, i.e. $Z_{s}\left(i_{a}, j_{a}\right)$ $=Z\left(i_{a}, j_{a}\right), a=1,2, \cdots, n$. For the first iteration, we add the differences $Z\left(i_{a}, j_{a} \square \Xi_{\mathrm{s}}\left(i_{a}, j_{a}\right)\right.$ to all simulated points close to $Z\left(i_{a}, j_{a}\right)$. This spread of the correction avoids discontinuity around the conditioned points.

Step 4: FFT is applied to the new image to obtain a new 
pair of amplitude and phase maps. Discard the new amplitude map, and inverse FFT is applied to the original amplitude and the new phase map, to obtain a new image of resource map. Repeating steps 3 and 4 until the value of the objective function is less than $o b j_{\min }$.

All the iteration processes use FFT and inverse FFT. Each time the simulated values are replaced to conditional data, so obj will certainly decline. Therefore, it can be expected that the process converges quickly until the density of object frequency spectrum complies with the conditional data. This simulation result considers the geological factors (hydrocarbon-bearing probability), and characterize the spatial distribution of discovered and undiscovered resource. It can be deemed as the desired prediction results.

\section{Application}

The Napu Sag, Bohai Bay Basin, China is taken as an application example. The Nanpu Sag, located in the northmost region of the Bohai Bay basin between Tangshan and Qinhuangdao, is a small petroliferous region with an area of $2,462 \mathrm{~km}^{2}$ (Fig. 1). The methods mentioned above are applied to predict the exploration risk and simulate the spatial distribution of resources in the 1st Member of Dongying Formation $\left(\mathrm{Ed}_{1}\right)$ of the Tertiary succession in the Nanpu Sag of the Bohai Bay Basin. For confidential reasons, all the data used in this study are the exploration results and seismic interpretations before the end of 2004 and the actual abundance was processed properly in the premise

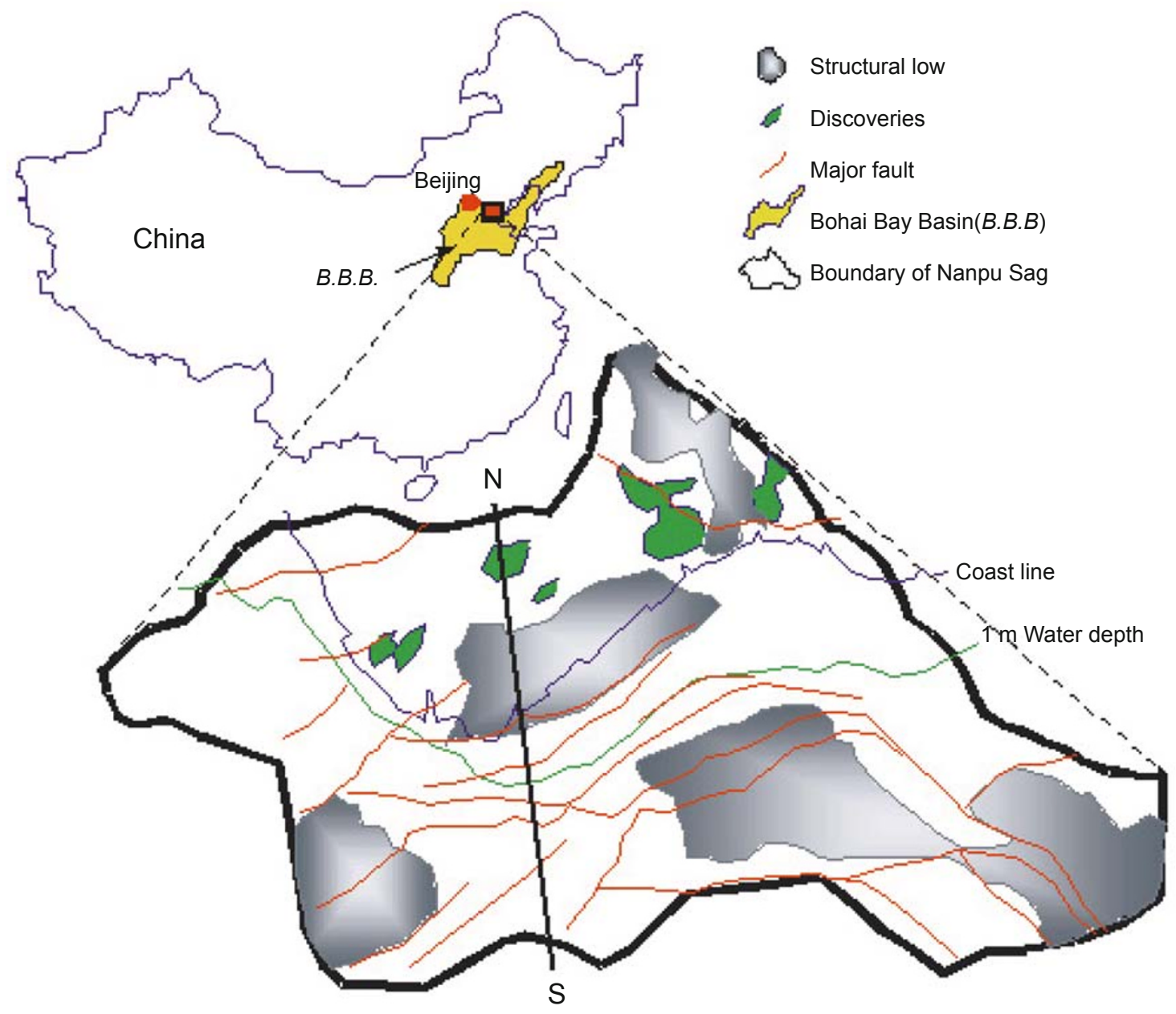

Fig. 1 Location of the Nanpu Sag

that the application of the methods is not affected. Up to the end of 2004, four oil fields, including Gaoshangpu, Liuzan, Laoyemiao and Beipu, were found, and total 224 exploration wells were drilled to or through $\mathrm{Ed}_{1}$, including 34 oil wells and 190 dry holes (Fig. 2).

\subsection{Simulation procedures}

(1) Mahalanobis distance and Fuzzy mathematics is used to integrate the seismic information and geological information, including $\mathrm{Ed}_{1}$ top structure, relative structure, distribution index of fault, bed thickness, content of sand, sand thickness, cap rock thickness of Guantao Formation, oil generation potential and oil drain potential of $\mathrm{Ed}_{1}$.

(2) A Bayesian formula is used to calculate the hydrocarbon-bearing probability, get the probability templates by Mahalanobis distance and Fuzzy mathematics, and predict the probability of exploration risk in the area (Fig. 3).

(3) Based on the results revealed by the 34 oil wells 
and corresponding $\mathrm{Ed}_{1}$ resource abundance, transform the discovered reservoirs in the study area to a hydrocarbon abundance map (denoted as HCMAP) (Fig. 4). The values at the pixel points are the average abundance of the discovered hydrocarbon accumulations at the study fields and zero at the dry hole locations and their influencing zones.

(4) HCMAP is transformed to a frequency domain by FFT, and an amplitude spectrum(denoted as OAMAP) and a phase spectrum (denoted as OPMAP) are obtained.

(5) Study the anisotropy of the OAMAP and use the fractal model to correct the sampling bias, and to obtain a modified amplitude map (denoted as MFAMAP).

(6) Integrate the geological information of the study area and to obtain a hydrocarbon-bearing probability map (Fig. 3 ) using the methods reported by $\mathrm{Hu}$ et al (2007) or Chen and Osadetz (2006a). Transform the probability map into the frequency domain to obtain an amplitude map and a phase map (denoted as GPMAP). We are particularly interested in the phase map because it contains conditional information about undiscovered petroleum accumulations.

(7) Perform the simulation procedures described above using the modified amplitude map MFAMAP and the phase

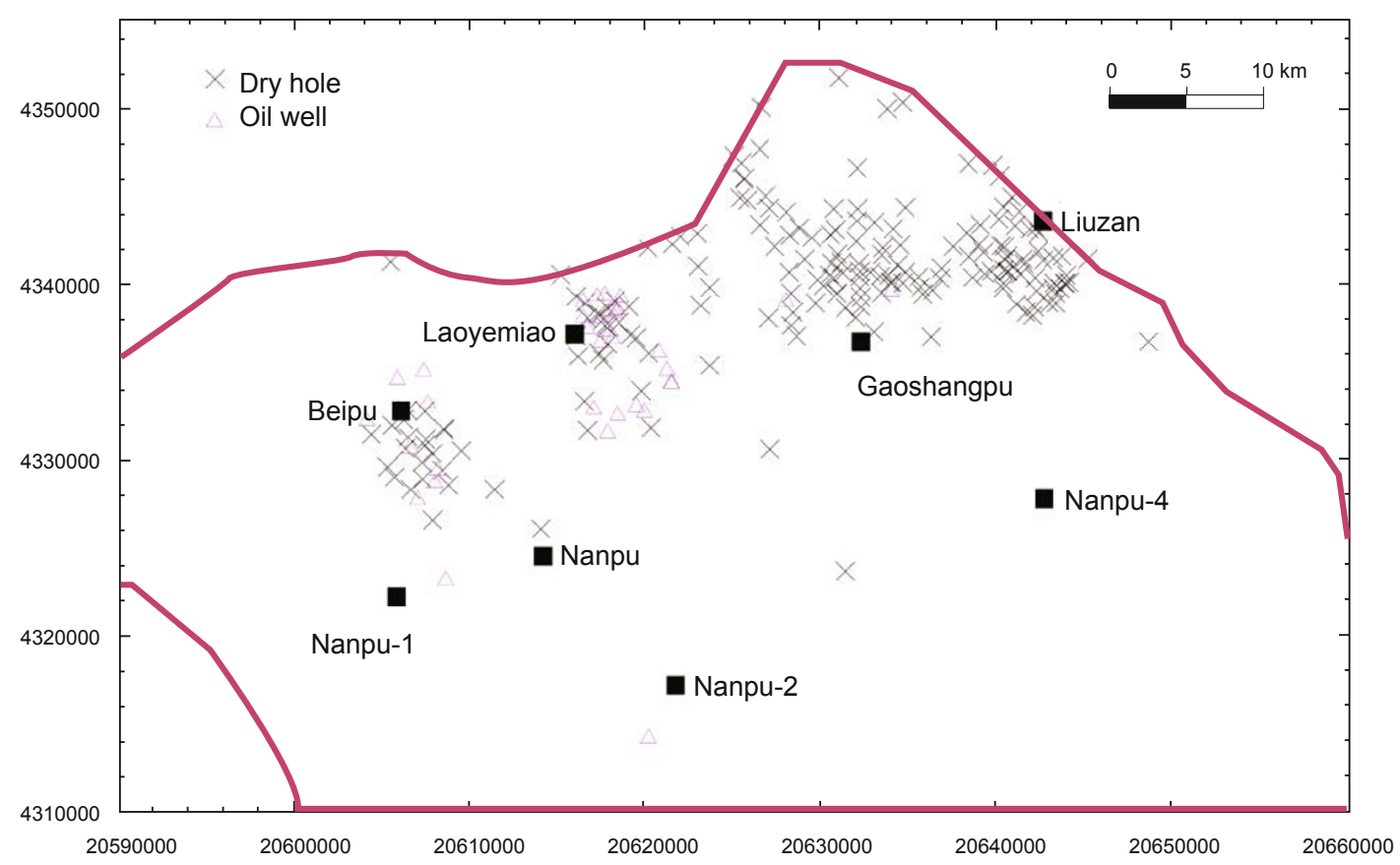

Fig. 2 Distribution of exploration wells in $\mathrm{Ed}_{1}$ in the Nanpu Sag

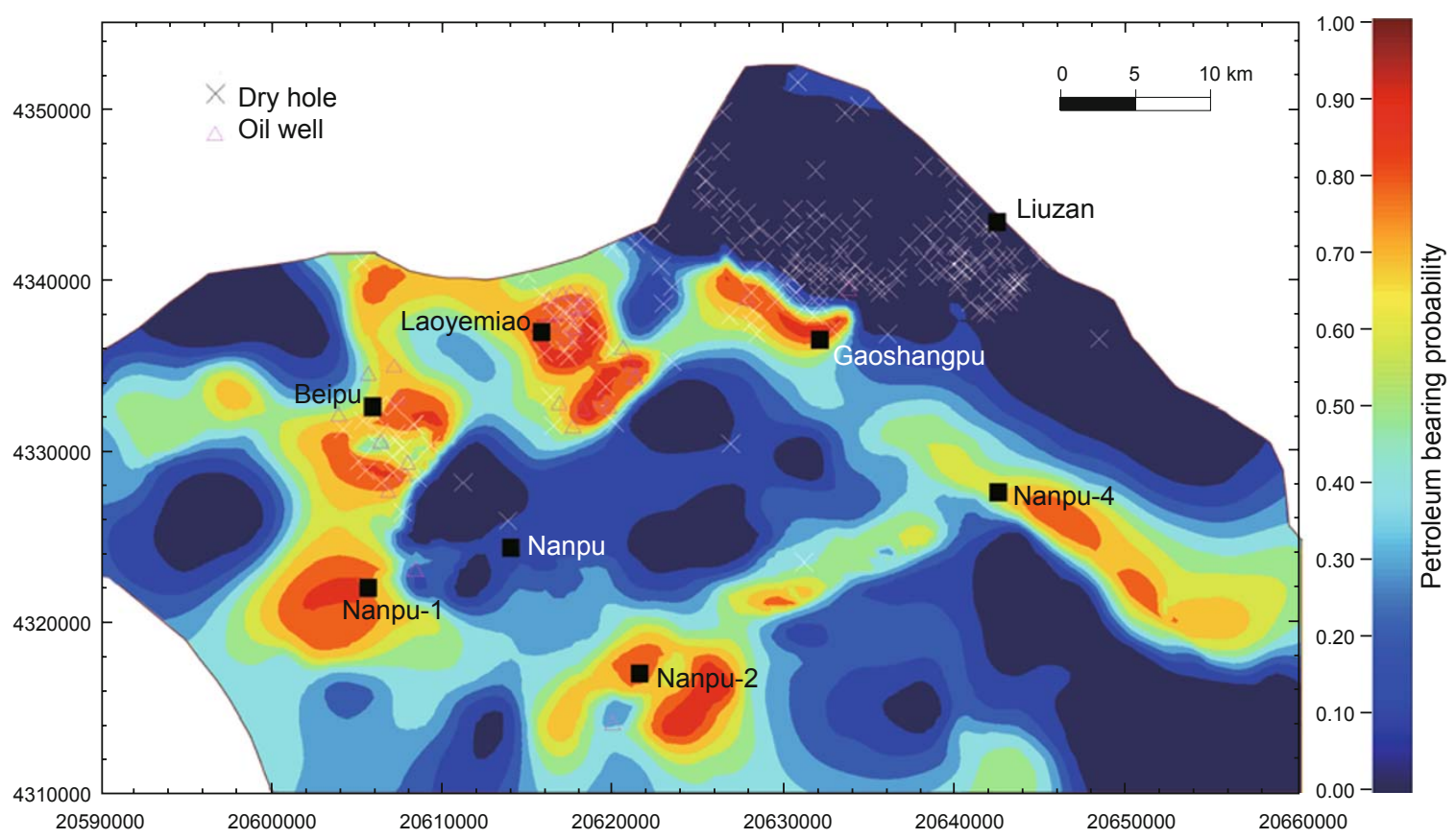

Fig. 3 Map of hydrocarbon probability in $\mathrm{Ed}_{1}$ 


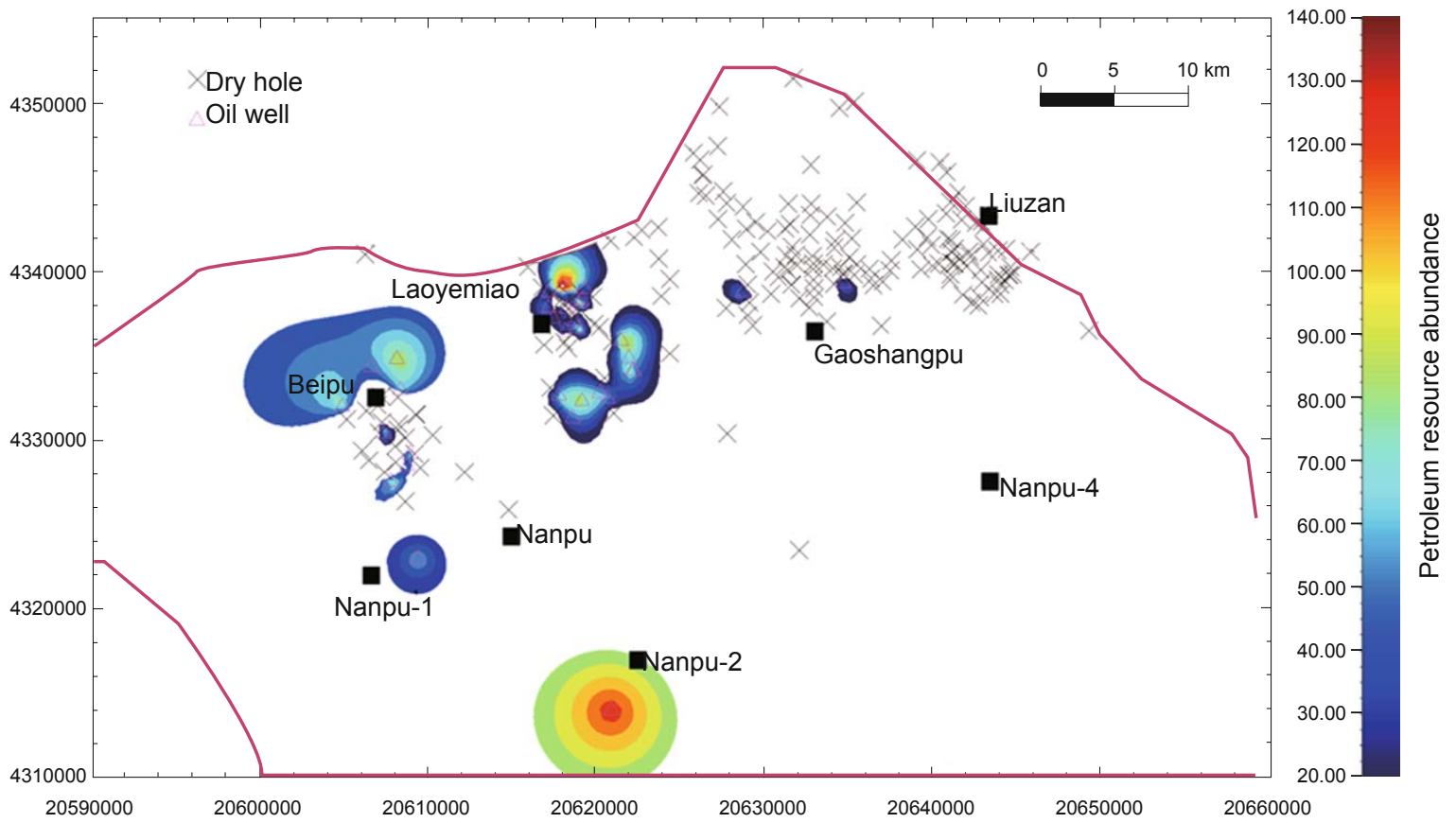

Fig. 4 Map of discovered hydrocarbon abundance (at 2004) in $\mathrm{Ed}_{1}$

map GPMAP from the probability map as input and the HCMAP as the conditioning data, to obtain a petroleum abundance map (denoted as MFHCMAP) (Fig. 5). This map represents the spatial distribution of the discovered and undiscovered petroleum accumulations in the study area.

\subsection{Simulation results}

Comparison of the predicted results with the actual exploration data shows that, in well explored areas in the northern sag (Gaoshangpu, Laoyemiao and Beipu fields), the $\mathrm{Ed}_{1}$ prediction results agree well with the actual exploration data; in the less explored areas in the southern sag, some abundant targets (including Nanpu-1, Nanpu-2 and Nanpu-4) are identified. In particular, the Nanpu-1 block with an area of $58.4 \mathrm{~km}^{2}$ shows an average abundance of $1.06 \times 10^{6}$ tonnes/ $\mathrm{km}^{2}$ and the estimated resources potential of $61.7 \times 10^{6}$ tonnes; Nanpu-2 block with an area of $92.8 \mathrm{~km}^{2}$ shows an average abundance of $1.53 \times 10^{6}$ tonnes $/ \mathrm{km}^{2}$ and the estimated resource potential of $14 \times 10^{6}$ tonnes; Nanpu- 4 block with an area of $36.8 \mathrm{~km}^{2}$ shows an average abundance of $1.3 \times 10^{6}$ tonnes/ $\mathrm{km}^{2}$ and the estimated resource potential of $48 \times 10^{6}$ tonnes. The prediction provides a useful guidance for exploration

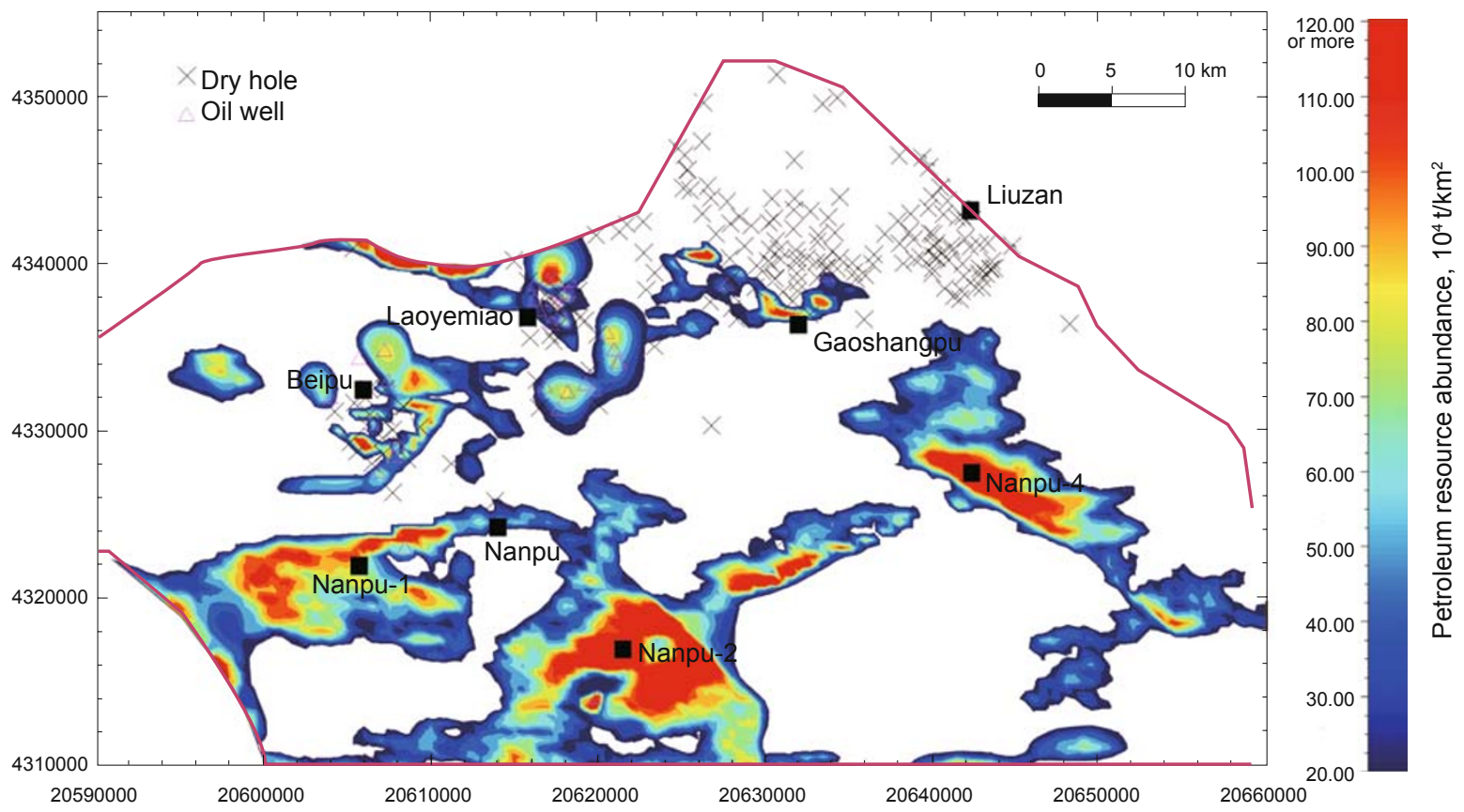

Fig. 5 Map of predicted hydrocarbon abundance in $\mathrm{Ed}_{1}$ 
decision-making.

The exploration results announced by PetroChina in May 2007 revealed big discoveries in Nanpu-1 and Nanpu-2.

\section{Conclusions}

The new prediction technology of petroleum spatial distribution discussed in this paper is based on the understanding and assumption of fractal characteristics of reservoirs and taking the Fourier integral approach. The simulation is performed through FFT and it is fast. The simulation gives not only the size of undiscovered petroleum accumulations, but also their possible locations.

The application to the Nanpu $\mathrm{Ed}_{1}$ shows that the fractal model captures the essential characteristics of petroleum distribution in space and the use of the exploration risk probability map in conditional simulation significantly improves the prediction. The simulation can be used as a tool for improve the exploration efficiency and success.

\section{References}

Barton C C and Scholz C H. The fractal size and spatial distribution of hydrocarbon accumulations. Fractal in petroleum geology and earth processes. New York: Plenum Press. 1995. 13-34

Chen $\mathrm{Z} \mathrm{H}$, Osadetz K G, Gao H, et al. Improving exploration success through uncertainty mapping, Keg River reef play, Western Canada Sedimentary Basin. Bulletin of Canadian Petroleum Geology. 2001. 49(3): 367-375

Chen Z H and Osadetz K G. Geological risk mapping and prospect evaluation using multivariate and Bayesian methods, western Sverdrup Basin of Canada. AAPG Bulletin. 2006a. 90(6): 859-87

Chen Z H and Osadetz K G. Undiscovered petroleum accumulation mapping using model-based stochastic simulation. Mathematical Geology. 2006b. 38(1): 1-16

Chen Z H, Osadetz K G and Hannigan P. Simulating the spatial distribution of undiscovered petroleum accumulations. Canadian International Petroleum Conference. 2002. Paper 2002-118, in the proceedings $\mathrm{CD}$

Gao H, Chen Z H, Osadets K G, et al. A pool based model of the spatial distribution of undiscovered petroleum resources. Mathematical Geology. 2000. 32: 7251-7749
Guo Q L, Wu N, Kong F Z, et al. Oil and gas exploration risk evaluation and screening of favorable areas for future exploration in Shenxian Sag, Bohai Bay Basin, China. Proceedings of IAMG 2007. 2007. 624-628

Guo Q L, Xie H B, Mi S Y, et al. Fractal model for petroleum resource distribution and its application. Acta Petrolei Sinica. 2009. 30(3): 379-385 (in Chinese)

Hu S Y, Guo Q L, Chen Z H, et al. A method of predicting petroleum resource spatial distribution and its application. Petroleum Exploration and Development. 2007. 34(1): 113-117 (in Chinese)

Hu S Y, Guo Q L, Chen Z H, et al. Probability mapping of petroleum occurrence with a multivariate-Bayesian approach for risk reduction in exploration, Nanpu Sag of Bohay Bay Basin, China. Geologos. 2009. 15(2): 91-102

Kaufman G M, Balcer Y and Kruyt D. A probabilistic model of oil and gas discovery, in Haun J (Ed), Estimating the volume of undiscovered oil and gas resources. AAPG Studies in Geology v. 1. 1975. 113-142

La Pointe P R. Estimation of undiscovered hydrocarbon potential through fractal geometry. Fractal in petroleum geology and earth processes. New York: Plenum Press. 1995. 35-57

Saupe D. Algarithms for random fractals, in: Pei-gen H O and Saupe D (Eds), The Science of Fractal Images. Springer-Verlag, New York. 1988. 71-136

Schuenemeyer J H and Drew L J. A procedure to estimate the parent population of the size of oil and gas fields as revealed by a study of economic truncation. Math. Geol. 1983. 15(1): 145-162

Turcotte D L. Fractals and Chaos in Geology and Geophysics. 2nd edition. Cambridge University Press. 1997

Xie H B, Guo Q L, Chen Z H, et al. Fractal conditional simulation of petroleum resource abundance and geographical location, Nanpu Sag of the Bohai Bay Basin, China. Proceedings of IAMG' 2007. 2007. 629-633

Yao T T. Conditional spectral simulation with phase identification. Mathematical Geology. 1998a. 30(3): 285-308

Yao T T. SPECSIM: a Fortran-77 program for conditional spectral simulation in 3D. Computers \& Geosciences. 1998b. 24(10): 911921

Zhao W Z, Hu S Y, Shen C X, et al. New advancements of oil and gas resource assessment methods. Acta Petrolei Sinica. 2005. 26 (Supplement): 25-29 (in Chinese)

(Edited by Zhu Xiuqin) 